\title{
Desempenho e satisfação ocupacional durante a prática do bodyboarding adaptado para pessoas com deficiências motoras: perspectivas de instrutores e praticantes ${ }^{1}$
}

\author{
Desempeño y satisfacción ocupacional durante la práctica de \\ bodyboard adaptado para personas con discapacidades motoras: \\ perspectivas de instructores y practicantes
}

Performance and occupational satisfaction during the practice of bodyboard adapted to people with motor deficiencies: perspectives from instructors and practitioners

\author{
Carla Lorena Passos Teles Roriz ${ }^{2}$ \\ Larissa Galvão da Silva ${ }^{3}$ \\ Aristela de Freitas Zanona ${ }^{4}$
}

Recibido: 12 de marzo 2019 • Enviado para modificación: 27 de julio 2019 • Aceptado: 16 de agosto 2019 Roriz, C.L.P.T., da Silva, L.G.. \& Zanona, A.F. (2019). Desempenho e satisfação ocupacional durante a prática do bodyboarding adaptado para pessoas com deficiências motoras: perspectivas de instrutores e praticantes. Revista Ocupación Humana, 19 (1), 37-49. doi: https://doi.org/10.25214/25907816.272

\section{RESUMO}

O estudo tem como objetivo investigar se a tecnologia assistiva universal existente para a prática do bodyboarding adaptado é suficiente para promover o desempenho ocupacional satisfatório de pessoas com deficiência motora. Foi realizada uma pesquisa quantitativa, transversal, com amostra selecionada por conveniência. Foi utilizado o Mini-Mental State Examination para

\footnotetext{
${ }^{1}$ Este artículo se deriva del trabajo de finalización de curso de la estudiante Carla Lorena Passos Teles Roriz, orientado por la docente Aristela de Freitas Zanona, en la Universidad Federal de Sergipe, Campus Antonio Garcia Filho, Brasil.

${ }^{2}$ Estudiante de Terapia Ocupacional, Universidad Federal de Sergipe, Campus Antonio Garcia Filho. Lagarto, Sergipe, Brasil. carla.Iorena_roriz@hotmail.com. ID ORCID: https://orcid.org/0000-00027499-3370

${ }^{3}$ Terapeuta ocupacional. Especialista en Terapia de Mano y Rehabilitación de Miembro Superior. Magíster en Terapia Ocupacional. Docente, Universidad Federal de Sergipe, Campus Antonio Garcia Filho. Lagarto, Sergipe, Brasil. larissagalvao.to@gmail.com. ID ORCID: https://orcid.org/0000-00026154-666X

${ }^{4}$ Terapeuta ocupacional. Especialista en Terapia Ocupacional con Enfoque Dinámico en Neurología. Magíster en Educación Física. Doctoranda en Neurociencias, Universidad Federal de Pernambuco. Docente, Universidad Federal de Sergipe, Campus Antonio Garcia Filho. Lagarto, Sergipe, Brasil. arisz_to@yahoo.com.br. ID ORCID: https://orcid.org/0000-0003-0684-2092
} 
selecionar instrutores e professionais. Para a variável de desempenho ocupacional, se utilizou a Medida Canadense de Desempenho Ocupacional. Participaram vinte sujeitos, doze instrutores e oito praticantes de bodyboard adaptado. Nos resultados do item grau de importância, para executar as atividades-problema levantadas pelos dois grupos, o grupo de instrutores obteve média de $9,83 \pm 0,25$, enquanto que o grupo de praticantes obteve $8,46 \pm 1,84$. O domínio desempenho ocupacional do grupo de instrutores apresentou médias de $6,35 \pm 1,97$, enquanto que, nesta mesma variável, o grupo de praticantes identificou a forma como executava a prática do

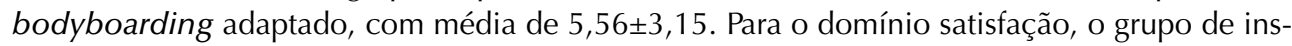
trutores apresentou média de $6,47 \pm 2,40$ e o grupo de praticantes de $6,63 \pm 3,60$. Conclui-se que os recursos adaptativos devem ser feitos sob medida e personalizados, visando corresponder à multiplicidade de fatores da pessoa com deficiência.

\title{
PALAVRAS-CHAVE
}

desempenho atlético, equipamentos de autoajuda, equipamento esportivo, esportes para pessoas com deficiência, Terapia Ocupacional

\section{RESUMEN}

El objetivo del estudio fue indagar si la tecnología universal de asistencia, existente para la práctica de bodyboard adaptado, es suficiente para promover el desempeño satisfactorio de personas con discapacidades. Se realizó una encuesta transversal cuantitativa, con muestra seleccionada por conveniencia. Se utilizó el Mini-Mental State Examination para seleccionar instructores y profesionales. Para la variable desempeño ocupacional se empleó la Medida Canadiense del Desempeño Ocupacional. Participaron veinte sujetos, doce instructores y ocho practicantes de bodyboard adaptado. En el ítem grado de importancia para realizar las actividades problemáticas, planteadas por ambos grupos, el grupo de instructores obtuvo un promedio de $9.83 \pm 0.25$, mientras en el grupo de participantes fue $8.46 \pm 1.84$. El dominio desempeño ocupacional en instructores presentó medias de $6.35 \pm 1.97$; en esa misma variable, los practicantes identificaron su forma de practicar el bodyboard adaptado con una media de $5.56 \pm 3.15$. Para el dominio satisfacción, el grupo de instructores tuvo una media de $6.47 \pm 2.40$ y el de practicantes, de $6.63 \pm 3.60$. Se concluye que los recursos adaptativos deben ser hechos a la medida y personalizados, para que coincidan con la multiplicidad de factores de la persona con discapacidad.

\section{PALABRAS CLAVE}

rendimiento atlético, dispositivos de autoayuda, equipamiento deportivo, deportes para personas con discapacidad, Terapia Ocupacional

\begin{abstract}
The study aims to investigate whether the existing universal assistive technology for the practice of adapted bodyboarding is adequate to promote the satisfactory occupational performance of people with motor disabilities. A quantitative, cross-sectional survey with convenience-selected sample was conducted. The occupational performance variable was measured using the Canadian Occupational Performance Measurement, and the Mini-Mental State Examination was used as a way to select instructors and practitioners. The study sample consisted of 20 subjects: 12 instructors and 8 participants of adapted bodyboarding.
\end{abstract}


The results in the item degree of importance to perform the problem, raised by both groups, the instructors group obtained an average of $9.83 \pm 0.25$, while the group of participants $8.46 \pm 1.84$. The domain occupational performance of the instructors showed medians of $6.35 \pm 1.97$, while in this same variable the group of participants identified the way they performed the practice of adapted bodyboarding with a median of $5.56 \pm 3.15$. For the domain satisfaction, the group of instructors presented a median of $6.47 \pm 2.40$ and the group of participants, $6.63 \pm 3.60$. It was concluded that adaptive resources must be tailor-made and personalized to match the multiple factors in the disabled person.

\section{KEYWORDS}

athletic performance, self-help devices, sports equipment, sports for disabled people, Occupational Therapy

\section{Introdução}

A prática esportiva é, historicamente, discutida como ferramenta relevante para a saúde. Os benefícios funcionais, físicos, psicológicos e socioculturais que o esporte proporciona justificam a crescente inclusão dessas práticas como forma terapêutica, recreativa, educativa e competitiva para pessoas com alguma deficiência (Marques, Castro \& Silva, 2001; Silva et al., 2013). O esporte adaptado permite um melhor desempenho dos atletas, além de propiciar momentos de lazer (Cardoso, 2011). Nesse sentido, dentre as diversas ocupações vivenciadas por um indivíduo, o lazer caracteriza-se como uma atividade não obrigatória, intrinsecamente motivada e realizada durante o tempo livre. O lazer/prática de esporte é uma excelente ferramenta para promover a satisfatória participação social, ou seja, maximizar a inter-relação de ocupações para apoiar o envolvimento desejado em atividades comunitárias e familiares, bem como facilitar o envolvimento em um subconjunto de atividades que envolvem situações sociais de suporte (American Occupational Therapy Association -AOTA, 2015).
Dentre todas as modalidades esportivas as aquáticas apresentam grande aceitação por parte das pessoas com deficiência. Diversos são os benefícios no corpo humano gerados pelas atividades realizadas na água: redução do tônus muscular, tornando os movimentos mais hábeis; início de movimentos (devido a flutuabilidade que a água oferece); redução de dor, de sintomas depressivos e estresse (Getz, Hutzler \& Vermeer, 2006; Krops et al., 2018). O surfing é um dos tipos de esportes aquáticos, que contribui de forma significativa para o desenvolvimento motor de pessoas com deficiências (Cordeiro, 2010).

Proveniente do surfing, o bodyboarding é um dos esportes que mais se desenvolveu nos últimos 10 anos. Ele foi considerado como a modalidade aquática que teve maior crescimento mundial no mercado (Mecías \& Navarro, 2015). O bodyboarding teve início no Havaí e, em seguida, foi oficializado como esporte na Califórnia. Atualmente a modalidade é praticada em cerca de 30 países, tais como Brasil, Chile, Venezuela, Peru, Espanha, Estados Unidos, França, Austrália, Marrocos e África do Sul. O esporte é pratica- 
do utilizando uma prancha de material esponjoso (39 a 42 polegadas), para que o surfista, posicionado em decúbito ventral, deslize sobre a superfície das ondas do mar. O tamanho da prancha dependerá da altura e peso de cada atleta, também é utilizado o pé-de-pato para realizar as manobras, impulsionando o atleta nas ondas (Scheffer, 2010). A prática desse esporte exige do atleta controle de tronco, com manutenção da cabeça elevada, bem como a contração isométrica de toda ou quase toda a musculatura de membros superiores e tronco. Os membros inferiores também são utilizados para impulsionar a prancha na água.

O bodyboarding adaptado surgiu da necessidade da prática de atletas com deficiência, especialmente motora, como lesão medular, paralisia cerebral, dentre outras, com o intuito de melhorar o desempenho nas ondas. Para isso, são necessários equipamentos com características particulares nessa modalidade. Esses equipamentos consistem no tamanho da prancha, relacionando-se com o peso e o tamanho do praticante (também chamada de prancha double); nadadeiras são menores e podem ter uma composição mais maleável; fator isotérmico fabricado com material Neoprene, com função de proteger o praticante do frio; shop ou leash (equipamento que fixa o praticante na prancha) e a parafina, que proporciona maior aderência entre a prancha e o praticante (Amado, 2011).

O termo esporte adaptado é utilizado apenas no Brasil e refere-se à prática do esporte por pessoas com deficiências, podendo ser necessárias adaptações nas estruturas esportivas ou na forma de execução do jogo para a possibilidade da prática. Assim, cada modalidade irá direcionar o planejamento e o desempenho do indivíduo durante sua realização (Silva, Marques \& Pena, et al, 2013). Para grande parte dos esportes adaptados, a tecnologia de assistência empregada é universal, ou também denominada de universal design. Contudo, a eficácia de tais recursos pode ser questionada, visto a multiplicidade de fatores que um indivíduo apresenta.

Desta forma, esse estudo tem como objetivo investigar se a tecnologia assistiva universal, existente para a prática do bodyboarding adaptado, é suficiente para promover o desempenho ocupacional satisfatório de pessoas com deficiência motora nessa prática esportiva.

\section{Material e método}

\section{Tipo de estudo}

O presente estudo caracteriza-se como uma pesquisa quantitativa. A amostra foi selecionada por conveniência e, por este motivo, não houve cálculo amostral. O delineamento da pesquisa foi transversal, avaliando os problemas de desempenho ocupacional satisfatório de pessoas com deficiência motora.

A pesquisa foi realizada nas instalações do projeto filantrópico Estrelas do Mar, em Aracaju/SE. O projeto teve início em março de 2011, através do bodyboarder Byron Silva. Surgiu da necessidade pessoal de proporcionar as pessoas com deficiência (motoras, intelectuais ou cognitivas) a possibili- 
dade de aprender e de praticar o esporte. As aulas ocorreram aos sábados e o projeto recebeu pessoas com todos os tipos de deficiência. As atividades programadas incluíram um momento lúdi$\mathrm{co}$, alongamento e aquecimento (técnicas realizadas ao ar livre, por meio de profissionais como: educador físico, terapeuta ocupacional, enfermeiro, psicólogo e fisioterapeutas); técnica de bodyboarding (orientações com aplicações de aulas teóricas e apresentações de vídeo-aulas por professores de educação física e bodyboarders); e a prática de bodyboarding (cada instrutor recebeu uma prancha para acompanhar o praticante). Os instrutores do bodyboarding são praticantes veteranos no esporte ou profissionais da educação física.

A pesquisa foi executada no período de novembro de 2018 a fevereiro de 2019, com quatro encontros. Neles, questionários foram aplicados para levantamento dos dados.

\section{Variáveis do estudo e instrumento de avalição}

A variável do desempenho ocupacional foi mensurada através do instrumento Medida Canadense de Desempenho Ocupacional (COPM). Tal instrumento consiste em uma medida individual de autopercepção do sujeito sobre os problemas encontrados no seu próprio desempenho ocupacional. A COPM foi publicada pela primeira vez em 1990 e traduzida para 24 idiomas, sendo exercida em mais de 35 países. A COPM é usada para: identificar áreas-problemas no desempenho ocupacional; oferecer uma quantificação das prioridades de desempenho ocupacional do sujeito; avaliar o desempenho e satisfação relacionados as áreas-problema; e medir as mudanças na autopercepção do cliente sobre o seu desempenho ocupacional ao longo do programa de intervenção de Terapia Ocupacional (Law et al., 2009).

O primeiro passo da entrevista é solicitar que o entrevistado identifique as ocupações em que ele apresenta dificuldade/limitações para executar. Uma vez identificadas; o sujeito classifica a importância daquelas ocupações em sua vida. A importância é pontuada em uma escala de 10 pontos, em que $1=$ "sem nenhuma importância" e 10= "extremante importante" (Law et al., 2009).

Em seguida, solicita-se que o entrevistado escolha até cinco problemas considerados como mais imediatos e importantes de serem resolvidos. Para estes cinco problemas, o sujeito pontua como ele considera seu próprio desempenho (também de 1 a 10, sendo $1=$ "incapaz de fazer" e $10=$ "capaz de fazer extremamente bem"). Além disso, pontua sua satisfação com seu desempenho naqueles cinco problemas escolhidos ("a pontuação irá de 1 a 10, em que 1 significa "nada satisfeito", e 10 "extremamente satisfeito com o desempenho"). Em seguida, essas pontuações são somadas e divididas pelo número de problemas, para gerar o escore total de desempenho. Desse mesmo modo, somam-se as pontuações da satisfação e as divide pelos problemas selecionados, para gerar o escore total da satisfação. Esses escores podem variar de 1 a 10 (Law et al., 2009).

O Mini-Mental State Examination (MMSE) foi utilizado como forma de selecionar os instrutores e praticantes, a fim de excluir da pesquisa aquele com 
déficit cognitivo acentuado (escore $\leq 4$ ). O teste é utilizado para avaliar a função cognitiva por ser rápido (em torno de 10 minutos), de fácil aplicação, não requerendo material específico. Deve ser utilizado como instrumento de rastreamento - não substituíndo uma avaliação mais detalhada -, pois apesar de avaliar vários domínios (orientação espacial, temporal, memória imediata e de evocação, cálculo, linguagem-nomeação, repetição, compreensão, escrita e cópia de desenho), não serve como teste de diagnóstico, mas sim para indicar funções (Folstein,Folstein \& Mchugh, 1975).

O primeiro passo para aplicar esse teste consiste na identificação do sujeito (nome, data nascimento e escolaridade). Os domínios são: orientação temporal, pontuando 1 para cada resposta correta, máximo de 10 pontos; registros, pontuando até 3 pontos; atenção e cálculo, com pontuação máxima até 5 pontos; lembranças ou memória de evocação, com pontuação máxima de 3 pontos; e linguagem com pontuação máxima de 9 pontos. A avaliação do escore obtido deve ser de acordo com os pontos de corte: 20 pontos para analfabetos; 25 pontos para pessoas com quatro anos de estudo; 26,5 pontos para indivíduos com 5 anos a 8 anos de estudo; 28 pontos para aqueles com 9 a 11 anos de estudo; 29 pontos para aqueles com mais de 11 anos de estudo (Folstein,Folstein \& Mchugh, 1975).

\section{Amostra e critérios de elegibilidade}

Constituiu-se como amostra desse estudo sujeitos que compuseram o grupo de instrutores e grupo de praticantes do bodyboarding adaptado. Definiu-se que somente participariam da pesquisa aqueles sujeitos que preenchessem os critérios de inclusão e não se enquadrassem nos de exclusão.

Para critérios de inclusão do grupo de instrutores, cada participante obedeceu as seguintes exigências: participar da prática do desporto aquático bodyboarding há mais de um mês; estar familiarizado com a cadeira anfíbia e prancha double (prancha de tamanho maior para praticante com deficiência); ter tempo de ensino da prática superior a três meses; obter preparação em primeiros socorros; ter idade igual ou maior que 18 e menor ou igual a 60 anos; e que concordasse em participar da pesquisa assinando o Termo de Consentimento Livre e Esclarecido.

Foram excluídos da pesquisa aqueles com déficit cognitivo, avaliados pelo instrumento Mini-Mental State Examination (escore menor que 20), (Folstein, Folstein \& Mchugh, 1975). Foram excluídos os instrutores com alteração visual e histórico de abuso de álcool e outras drogas.

Para o grupo de praticantes, foram incluídos os participantes com idade entre 18 anos e 60 anos; acometidos por deficiência motora (mediante comprovação do CID 10 - Classificação Internacional de Doenças), com controle cervical; que participassem da prática desportiva aquática do bodyboarding a mais de um mês, apresentassem o cognitivo preservado, avaliado pelo Mini-Mental State Examination (escore maior que 20), (Folstein, Folstein \& Mchugh, 1975) e concordassem em participar da pesquisa, assinando o Termo de Consentimento Livre e Esclarecido. Para o grupo de praticantes, foram excluídos os com déficit cognitivo gra- 
ve, acometidos por múltiplas deficiências e com histórico de abuso de álcool e drogas.

\section{Fluxo da pesquisa}

Os instrumentos de avaliação supracitados foram aplicados por profissionais terapeutas ocupacionais treinados e calibrados no uso dos testes. Os entrevistados foram divididos em dois grupos: instrutores e praticantes. Nesse sentido, realizou-se a aplicação dos testes uma única vez, a fim de levantar problemas no desempenho e satisfação no desempenho ocupacional, como a tecnologia assistiva existente na prática do bodyboarding.

Para análise dos dados, foi utilizada a estatística descritiva com medidas de tendências central e dispersão (média, desvio padrão e porcentagem). Também foi utilizado o programa Excel para Windows do pacote do Microsoft 2010.

\section{Aspectos éticos}

A presente pesquisa foi aprovada pelo Comitê de Ética em Saúde da Universidade Federal de Sergipe sob o número da CAAE: 02644518.1.0000.5546.

Os participantes foram esclarecidos sobre os objetivos da pesquisa e informados sobre a ausência de riscos e benefícios de sua participação nela. Todos assinaram o Termo de Consentimento Livre e Esclarecido.

\section{Resultados}

O projeto conta com 30 instrutores e 44 praticantes, contudo, por não se enquadrarem nos critérios de inclusão, foram excluídos 18 instrutores e 36 praticantes. A amostra foi constituída por 20 participantes, sendo que 12 pertenciam ao grupo de instrutores e 8 ao grupo de praticantes. Na tabela 1, estão descritos os dados de caracterização dos sujeitos.

Tabela 1. Caracterização da amostra.

\begin{tabular}{|c|c|c|c|}
\hline \multicolumn{2}{|c|}{ Características } & $\begin{array}{l}\text { Grupo de } \\
\text { Instrutores }\end{array}$ & $\begin{array}{l}\text { Grupo de } \\
\text { Praticantes }\end{array}$ \\
\hline $\begin{array}{l}\text { Sexo } \\
(\%)\end{array}$ & $\begin{array}{l}\text { Feminino } \\
\text { Masculino }\end{array}$ & $\begin{array}{l}41,6 \\
58,4\end{array}$ & $\begin{array}{l}25 \\
75\end{array}$ \\
\hline \multicolumn{2}{|l|}{ Idade (anos) } & $25,2 \pm 7,1$ & $31,75 \pm 7,6$ \\
\hline $\begin{array}{l}\text { Escolaridade } \\
(\%)\end{array}$ & $\begin{array}{l}\text { Ensino infantil } \\
\text { Ensino fundamental } \\
\text { Ensino médio } \\
\text { Ensino superior }\end{array}$ & $\begin{array}{l}- \\
- \\
66,6 \\
33,4\end{array}$ & $\begin{array}{l}12,5 \\
25 \\
37,5 \\
25\end{array}$ \\
\hline \multicolumn{2}{|c|}{ Tempo de prática (meses) } & $57,9 \pm 53,2$ & - \\
\hline \multicolumn{2}{|c|}{ Tempo de ensino (meses) } & $38,0 \pm 29,2$ & - \\
\hline $\begin{array}{l}\text { Tipo de } \\
\text { deficiência } \\
(\%)\end{array}$ & $\begin{array}{l}\text { Adquirida } \\
\text { Congênita }\end{array}$ & - & $\begin{array}{l}12,5 \\
87,5\end{array}$ \\
\hline \multicolumn{2}{|c|}{ Tempo de deficiência (meses) } & - & $39,6 \pm 23,0$ \\
\hline
\end{tabular}

*Os dados estão representados como média, desvio padrão e porcentagem.

Fonte: elaboração própria. 
Na tabela 2, podem ser encontrados os problemas mais citados pelos instrutores e praticantes com relação à prática do esporte em si, à estrutura física\ acessibilidade da praia e dificuldades inerentes das deficiências motoras.
Com relação ao item grau de importância, para executar as atividades-problema levantadas pelos dois grupos, o grupo de instrutores obteve média de $9,83 \pm 0,25$, enquanto o grupo de praticantes obteve $8,46 \pm 1,84$.

Tabela 2. Problemas levantados para a prática do esporte por ambos os grupos durante aplicação da Medida Canadense de Desempenho Ocupacional.

\begin{tabular}{|c|c|c|}
\hline \multicolumn{2}{|r|}{ Problemas Levantados } & $\begin{array}{c}\text { Frequência } \\
(\%)\end{array}$ \\
\hline \multirow{15}{*}{$\begin{array}{l}\text { Grupo de } \\
\text { Instrutores }\end{array}$} & Peso do assistido & 41,6 \\
\hline & Tipo de deficiência & 41,6 \\
\hline & Deslocamento para o mar & 41,6 \\
\hline & Falta de equipamentos & 41,6 \\
\hline & Altura do assistido & 33,3 \\
\hline & Deficiência de profissionais & 33,3 \\
\hline & Estrutura local & 16,6 \\
\hline & Falta de cordas para suporte & 16,6 \\
\hline & Entrada no mar & 8,3 \\
\hline & Mar muito agitado & 8,3 \\
\hline & Rotatividade dos voluntários & 8,3 \\
\hline & Necessidade de mais voluntários & 8,3 \\
\hline & Medo por parte dos voluntários & 8,3 \\
\hline & Interação com os assistidos & 8,3 \\
\hline & Capacitação dos voluntários & 8,3 \\
\hline \multirow{10}{*}{$\begin{array}{l}\text { Grupo de } \\
\text { Praticantes }\end{array}$} & Dificuldade em relação ao tamanho da prancha & 66,5 \\
\hline & Dificuldade de transferência para a cadeira anfíbia & 50 \\
\hline & Tipo de deficiência & 50 \\
\hline & Dificuldade para realizar alongamentos & 37,5 \\
\hline & Dificuldade de deslocamento para o mar & 25 \\
\hline & Dificuldade em relação à altura & 25 \\
\hline & Dificuldade no equilíbrio & 25 \\
\hline & Dificuldade em relação ao peso & 12,5 \\
\hline & Dificuldade no transporte até o local & 12,5 \\
\hline & Falta de voluntários & 12,2 \\
\hline
\end{tabular}

Fonte: Elaboração própria.

Para o domínio desempenho ocupacional do grupo de instrutores (ou seja, como eles percebiam a forma do seu próprio ensino da prática), o grupo de instrutores apresentou média de
$6,35 \pm 1,97$, enquanto que, nessa mesma variável, o grupo de praticantes identificou a forma como executava a prática do bodyboarding adaptado com média de $5,56 \pm 3,15$. 
Para o domínio satisfação, o grupo de instrutores apresentou média de $6,47 \pm 2,40$ e o grupo de praticantes de $6,63 \pm 3,60$. O desempenho e a satisfação ocupacional, avaliados pela Medida Canadense de Desempenho Ocupacional, estão representados no gráfico 1 .
Após a análise dos resultados das avaliações padronizadas e da discussão com os integrantes, os terapeutas ocupacionais identificaram que os baixos escores relacionados à prática e à satisfação com a atividade, podem ser entendidos pela falta de acessibilidade

Gráfico 1. Grau de importância, desempenho e satisfação ocupacional avaliados pela Medida Canadense de Desempenho Ocupacional.

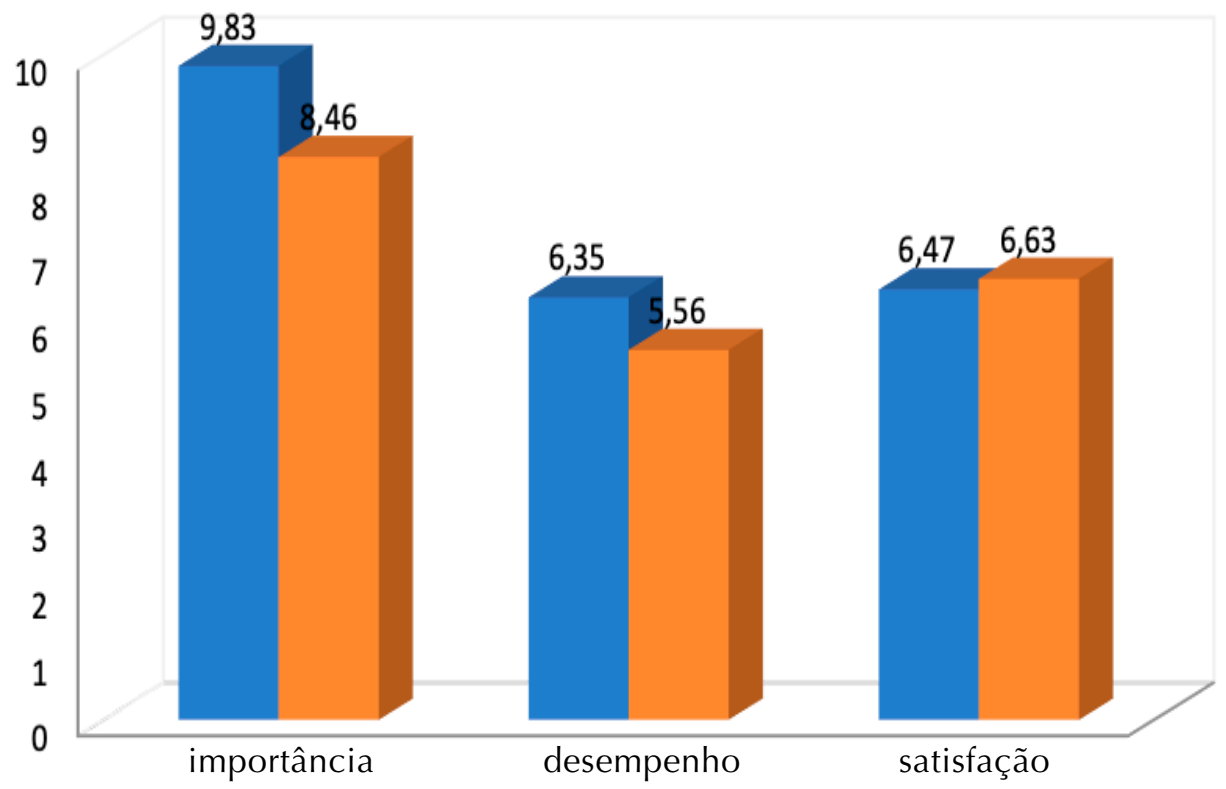

Fonte: tlaboraçāo própria.

do ambiente e pela falta de um planejamento individualizado para potencializar o desempenho no esporte. Todas as atividades executadas no projeto acabam não levando em conta as especificidades de cada sujeito, inclusive o uso do material e equipamentos - que já são considerados adaptativos, mas que não respondem as demandas específicas dos sujeitos com deficiência.

\section{Discussão}

Almejar ou manter a saúde é importante para qualquer pessoa. Neste sentido, é ainda mais importante para pessoas com deficiência, sendo maior o obstáculo enfrentado. Assim, para promover a prática desportiva para esse publico, é necessária uma abordagem diferente (Getz, Hutzler \& Vermeer, 2006; Mclo- 
ghlin, Weisman, Castaneda, Gwin \& Graver, 2017; Hoekstra et al., 2019).

Nos últimos anos, houve um grande aumento no uso de equipamentos adaptativos por pessoas com deficiência. Nesse aspecto, o uso da tecnologia assistiva torna-se fundamental e amplamente recomendado. Entretanto, o conhecimento acerca desse campo ainda é reduzido. Os equipamentos adaptativos utilizados em atividades físicas, esportes e lazer precisam ser melhor projetados, para permitir um desempenho ótimo do atleta (Bergem, 2019).

Da mesma forma que a relação do atleta com a prática do esporte é influenciada pela sua deficiência, os equipamentos envolvidos precisam ser moldados para cada tipo físico. Ademais, quanto maior a funcionalidade de uma adaptação, menor a chance de lesões e abandono da tecnologia, caso esta não corresponda às demandas esperadas do atleta. Essas relações, se não forem bem desenvolvidas, podem gerar danos, podendo se estender além da exclusão do esporte, como também limitando a independência funcional e a capacidade de executar a participação social (Dutton, 2019).

Segundo Ellis, Callaway e Dyer (2018), que realizaram pesquisa com a modalidade esportiva Paracanoagem, a adaptação no esporte aquático, deve estar de acordo com as necessidades específicas de cada atleta com deficiência, levando em consideração o seu conforto durante a prática.

Ao compararmos essa prática com a modalidade do bodyboarding, nota-se o quanto são necessárias adaptações personalizadas para a prática desportiva, visto os problemas levantados pelos praticantes e instrutores (tabela 2) e as notas de desempenho e satisfação que ambos os grupos avaliaram com relação ao ensino e a prática do esporte, respectivamente. Entretanto, por não haver tanta projeção no cenário de competições, a prática do bodyboarding não foi tão analisada para desenvolver adaptações suficientes para a performance do indivíduo. A inserção desse esporte na categoria de elite aumentaria a necessidade de estudos, consequentemente, melhoraria o desempenho e a satisfação do atleta.

A prática desportiva aquática para pessoas com deficiência enfrenta diferentes barreiras e uma abordagem diferente pode ser necessária para desenvolver o esporte adaptado. É relevante tratar dos recursos humanos, das instalações, dos materiais e equipamentos (Ramos \& Isayama, 2009). Nesse sentido, existem profissões especializadas na indicação e elaboração de tecnologias de assistência, tais como Fisioterapia e a Terapia Ocupacional. Por meio da lei $n^{\circ} 13.146$ de 2015 a Terapia Ocupacional orienta, prescreve, executa, desenvolve produtos, recursos, e estratégias para melhorar o desempenho de pessoas com deficiência nas diferentes áreas de ocupação (incluindo lazer e esporte) (Brasil, 2015).

O uso de adaptações proporciona um processo de reabilitação e soluciona, por vezes, problemas em ambientes segregados e exclusivos. Entretanto, os recursos, atualmente, não são suficientes para atingir as demandas. Sendo assim, os profissionais deparam-se com diversas dificuldades para desenvolver adaptações únicas e singulares para cada indivíduo, de acordo com seus objetivos e atribuições. Torna-se evidente, que as tecnologias assistivas não po- 
dem ser universais, ou "prontas" (Hutzler, 2007). A expertise do terapeuta ocupacional, para elaborar tecnologias de assistência personalizadas, deve ser cada vez mais aproveitada para promover a autonomia dessas pessoas com deficiência, não só para se engajarem na atividade esportiva, mas também para permitir uma melhor performance para demandas competitivas.

\section{Conclusão}

As adaptações proporcionam oportunidades para manter ou desenvolver funções corporais através da prática desportiva. A busca por uma melhor atuação no desempenho da prática do bodyboarding adaptado influencia diretamente no estado psíquico do indivíduo, melhorando ou diminuindo seu nível de satisfação. Desse modo, para garantir melhores resultados nessa modalidade desportiva, conclui-se que o uso de equipamentos deve ser personalizado, ou seja, de acordo com as necessidades e atribuições de cada praticante. A tecnologia assistiva universal existente, atualmente, para a prática do bodyboarding adaptado não é suficiente para promover o desempenho ocupacional satisfatório de pessoas com deficiência motora nessa prática desportiva.

Constituiu-se como limitações desse estudo a falta do cálculo amostral e a distribuição assimétrica e randomizada nos grupos. Além disso, as diferenças entre os tipos e tempo de deficiências (congênita e adquirida) podem gerar vieses com relação à percepção do desempenho. Desta forma, sugere-se que, em estudos futuros, essas variáveis possam ser melhor controladas.

\section{Referências}

Amado, D.F.L. (2011). Programa de desenvolvimento do bodyboard no Concelho da Nazaré. Estudo de viabilização da implementação do bodyboard como actividade extra curricular no Ext. Dom Fuas Roupinho (dissertação de mestrado). Universidad de Coimbra, Coimbra, Portugal.

American Occupational Therapy Association -AOTA. (2015). Estrutura da prática da Terapia Ocupacional: domínio \& processo - $3^{\text {a }}$ ed. traduzida. Revista de Terapia Ocupacional da Universidade De São Paulo, 26(esp), 1-49.

Bergem, S. (2019). Knowledge among important actors in the field of adaptive equipment for young people with disabilities. Disability and Rehabilitation: Assistive Technology, 1-10. doi: https://doi.org/10 .1080/17483107.2018.1538393

Brasil (2015). Lei Brasileira de Inclusão da Pessoa com Deficiência (Estatuto da Pessoa com Deficiência). Lei $n{ }^{\circ} 13.146,6$ de julho de 2015. Disponível em: http:// www.planalto.gov.br/ccivil_03/_Ato20152018/2015/Lei/L13146.htm

Cardoso, V.D.A. (2011). A Reabilitação de pessoas com deficiência através do desporto adaptado. Revista Brasileira de Ciências do Esporte, (33)2, 529-539.

Cordeiro, P.T. (2010). Ambiente e acessibilidade para pessoas com deficiência no desenvolvimento de atividades esportivas em piscinas: Um estudo de Caso (tese de mestrado). Centro Universitário Univates, Lajeado, Rio Grande do Sul, Brasil.

Dutton, R.A. (2019). Medical and Musculoskeletal Concerns for the Wheelchair Athlete: A Review of Preventative Strate- 
gies. Current Sports Medicine Reports, 18(1), 9-16. doi: https://doi.org/10.1249/ JSR.0000000000000560

Ellis, S., Callaway, A. \& Dyer, B. (2018). The influence of lower-limb prostheses technology on Paracanoeing time-trial performance. Disability and Rehabilitation: Assistive Technology, 13(6), 568-574. doi: https://doi.org/10.1080/17483107.20 17.1357052

Folstein, M.F., Folstein, S.E. \& Mchugh, P.R. (1975). Mini-Mental State: a practical method for grading the cognitive state of patients for clinicians. Journal Psychiatric Research, 12(3), 189-198.

Getz, M., Hutzler, Y. \& Vermeer, A. (2006). Effects of aquatic interventions in children with neuromotor impairments: a systematic review of the literature. Clinical Rehabilitation, 20(11), 927-936. doi: https:// doi.org/10.1177/0269215506070693

Hoekstra, F., Roberts, L., Van Lindert, C., Matin Ginis, K., van der Woude, L.H. V. \& McColl, M.A. (2019). National approaches to promote sports and physical activity in adults with disabilities: examples from the Netherlands and Canada. Disability and Rehabilitation, 41(10), 1217-1226. doi: https://doi.org/10.1080/09638288.2017.1 423402

Hutzler, Y. (2017). A Systematic Ecological Model for Adapting Physical Activities: Theoretical Foundations and Practical Examples. Adapted Physical Activity Quarterly, 24(4), 287-304. doi: https:// doi.org/10.1123/apaq.24.4.287

Krops, L.A., Hols, D.H.J., Folkertsma, N., Dijkstra, P., Geertzen, J.H.B. \& Dekker, R. (2018). Requirements on a communi- ty-based intervention for stimulating physical activity in physically disabled people: a focus group study amongst experts. Disability and Rehabilitation, 40(20), 2400-2407. doi: https://doi.org/10.1080/ 09638288.2017.1336645

Law, M., Baptiste, S., Carswell, A, McColl, M.A., Polatajko, H. \& Pollock, N. (2009). Medida Canadense de Desempenho Ocupacional (COPM) (Trad. Magalhães, L., Magalhães, L. \& Cardoso, A.). Belo Horizonte: Belo Horizonte: Editora Universidade Federal de Minas Gerais.

Marques, M.U., Castro, J.A.M. \& Silva, M.A. (2001). Actividade Física Adaptada: uma visão crítica. Revista Portuguesa de Ciências do Desporto. (1) 1,73-79.

Mcloughlin, G., Weisman, F.C., Castaneda, Y., Gwin, C. \& Graver, K. (2017). Sport participation for elite athletes with physical disabilities: motivations, barriers, and facilitators. Adapted Physical Activity Quarterly, 34(4), 421-41. doi: https://doi. org/10.1123/apaq.2016-0127

Mecías, M. \& Navarro, R. (2015). Bases teóricas del entrenamiento para jóvenes deportistas en deportes acuáticos en el médio natural: Bodyboard. Sportis. Scientific Journal of School Sport, Physical Education and Psychomotricity, (1)3, 345364. doi: https://doi.org/10.17979/sportis.2015.1.3.1422

Ramos, R. \& Isayama, H.F. (2009). Lazer e esporte: olhar dos professores de disciplinas esportivas do curso de educação física. Revista Brasileira de Educação Física e Esporte, (23)4, 379-91.

Scheffer, F. (2010). Um olhar sobre o bodyboarding a partir de uma revisão de lite- 
ratura (trabalho de conclusão de curso). Porto Alegre, Universidade Federal do Rio Grande do Sul, Brasil.

Silva, A.A.C., Marques, R.F.R., Pena, L.G.S., Molchansky, S., Borges, M., Campos, L.F.C.,... Gorla, J.I. (2013). Esporte adaptado: abordagem sobre os fatores que influenciam a prática do esporte coletivo em cadeira de rodas. Revista Brasileira de Educação Física e Esporte, 679-687. doi: http://dx.doi.org/10.1590/ S1807-55092013005000010 